\title{
SISTEMA AUTOMÁTICO DE DETECÇÃO E CLASSIFICAÇÃO DE DISTÚRBIOS ELÉTRICOS EM QUALIDADE DA ENERGIA ELÉTRICA
}

\author{
Danton Diego Ferreira* \\ danton@lps.ufrj.br \\ Cristiano Augusto Gomes Marques* \\ cristianodlps.ufrj.br
}

\author{
Augusto Santiago Cerqueira ${ }^{\dagger}$ \\ augusto.santiago@ufjf.edu.br \\ Carlos Augusto Duque ${ }^{\dagger}$ \\ carlos.duque@ufje.edu.br
}

\author{
Moisés Vidal Ribeiro ${ }^{\dagger}$ \\ moviribeiro@ieee.org \\ *Universidade Federal do Rio de Janeiro \\ COPPE/EP \\ Rio de Janeiro, RJ, Brasil \\ ${ }^{\dagger}$ Universidade Federal de Juiz de Fora \\ Labsel \\ Juiz de Fora, MG, Brasil
}

\begin{abstract}
This contribution outlines signal processing-based algorithms for the detection and classification of voltage disturbances in power system. Basically filtering technique is applied to decompose the voltage signal into two primitive components which are named fundamental and error ones, then higher-order statistics (HOS)-based feature are selected and applied to detect and classify disturbances. Bayesand Neural Network- based techniques are designed for the detection and classification respectively. The system was simulated considering six classes of disturbances, achieving a global efficiency about $100 \%$ to such disturbances. The performance of the method is compared with other methods presented in the literature.
\end{abstract}

KEYWORDS: Power Quality, Higher-Order Statistics, Artificial Neural Network.

\section{RESUMO}

Este trabalho apresenta um sistema de detecção e classificação de distúrbios de qualidade da energia elétrica (QEE) que se baseia na decomposição do sinal de tensão em dois novos sinais, referentes à componente fundamental e ao sinal de erro e, em seguida, utiliza Estatísticas de Ordem Superior (EOS) para extrair parâmetros representativos de cada classe para simplificar o algoritmo de detecção e classificação. Como detector é utilizado um algoritmo baseado na teoria de Bayes e para implementar o algoritmo de classificação utilizou-se uma rede neural artificial. O sistema foi testado em simulações para seis classes de distúrbios, apresentando uma eficiência global próxima a $100 \%$ para tais distúrbios. Os resultados aqui apresentados são comparados com os resultados de outros sistemas propostos na literatura.

PALAVRAS-CHAVE: Qualidade da Energia Elétrica, Estatísticas de Ordem Superior, Rede Neural Artificial.

Artigo submetido em 10/04/2007 (Id:868)

Revisado em 04/06/2008 e em 21/11/2008

Aceito sob recomendação do Editor Associado Prof. Eduardo N. Asada 


\section{INTRODUÇÃO}

O crescente uso de equipamentos computadorizados e sensíveis às perturbações do sistema elétrico tem exigido requisitos da qualidade da energia elétrica (QEE) cada vez mais rigorosos (Heydt, 1998). Ao mesmo tempo que cresce a exigência, por parte dos consumidores, de uma energia de melhor "qualidade", as concessionárias de energia elétrica, responsáveis pelo fornecimento desta energia, se vêem numa situação delicada. Pois o aumento do número de cargas de natureza não-linear (sistemas micro-processados, fontes chaveadas, etc) em instalações elétricas residenciais, comerciais e industriais provoca o aumento significativo da ocorrência de distúrbios nos seus sistemas de distribuição e a consequente perda da qualidade da energia elétrica entregue (Arrillaga et al., 2000). Como as agências reguladoras não definiram ainda regras mais restritivas, tanto para os consumidores, como para as concessionárias, em relação ao nível de "contaminação" que os primeiros podem injetar na rede e o nível de "qualidade" que as concessionárias devem manter, o cenário encontra-se aberto a batalhas judiciais, o que incentiva o aumento de pesquisas nesta área.

Cumpre ressaltar que organismos internacionais e nacionais estão trabalhando no sentido da definição de normas. No Brasil existe um esforço por parte do governo para a regulamentação dos padrões da qualidade de energia elétrica nos níveis de transmissão e distribuição. Atualmente, encontrase em fase de regulamentação o PRODIST: Procedimentos de Distribuição (ANEEL, 2008), que contém os documentos regulatórios que descrevem alguns indicadores de desempenho e de qualidade dos serviços de energia elétrica.

De maneira geral, os distúrbios da QEE (eventos), se manifestam como deformidades na forma de onda da tensão e corrente em um sistema elétrico de potência. Estas deformidades, freqüentemente referidas como problemas de QEE, afetam significativamente as indústrias em diversos aspectos (Arrillaga et al., 2000). Dentre eles, destacam-se a interrupção do processo de produção e baixa velocidade nas redes de computadores, o que é refletido em significativas perdas financeiras para as indústrias.

Neste contexto, o desenvolvimento e o aperfeiçoamento de sistemas de monitoramento da QEE, com destaque para a detecção e classificação de distúrbios, é fortemente justificada e constitui-se no objetivo deste trabalho.

A análise da QEE requer o uso de diversas ferramentas modernas de processamento de sinais. O trabalho de Oleskovicz et al. (2006) aparece como uma importante contribuição, uma vez que apresenta um estudo comparativo de ferramentas modernas de análise aplicadas à QEE.

Em um sistema de monitoramento da QEE, a detecção é o primeiro passo para uma correta análise do problema, uma vez que a correta detecção dos distúrbios facilita o emprego das técnicas de classificação, identificação e compressão de sinais, posto que o uso das mesmas deve ocorrer quando os distúrbios são detectados. Várias técnicas baseadas na transformada wavelet, FFT (Fast Fourier Transform), e inteligência computacional têm sido aplicadas diretamente na detecção de distúrbios em QEE (Ibrahim e Morcos, 2002; Poisson et al., 2000; Yang e Liao, 2001; Santoso et al., 2000; Oleskovicz et al., 2006). É importante mencionar também os trabalhos de Ramos et al. (2002), Ribeiro et al. (2007) e Duque et al. (2005) que apresentam uma diferente contribuição para detecção de eventos, com técnicas baseadas no princípio Dividir e Conquistar.

No campo da classificação, podemos citar os trabalhos de Wang e Mamishev (2004), Wang et al. (2004), Wang et al. (2001), Cerqueira et al. (2005), Abdel-Galil et al. (2004), He e Starzyk (2006) e Ferreira et al. (2006) que obtiveram taxas de classificação entre $90 \%$ e $100 \%$ para algumas classes de distúrbios elétricos em QEE. É importante destacar também o trabalho de Anil et al. (2000) que faz uma boa revisão apontando vantagens e desvantagens de algumas das principais técnicas de classificação.

Neste trabalho, um sistema de detecção e classificação de distúrbios em QEE é proposto. Este sistema é baseado na idéia da utilização de parâmetros de estatísticas de ordem superior (EOS) tanto para detectar quanto para classificar os distúrbios aqui abordados. Este tipo de abordagem é motivada pelo fato de as EOS serem imunes à presença de ruído gaussiano (Nikias e Petropulu, 1993) e foi, inicialmente, aplicada ao problema de detecção e classificação de distúrbios elétricos em Ribeiro et al. (2007) e Ferreira et al. (2006), resultando em um elevado desempenho aliado a uma reduzida complexidade computacional. As principais vantagens do sistema proposto neste trabalho são: $i$ ) uma maior imunidade a ruídos Gaussianos; $i$ ) a simplificação do algoritmo de detecção e classificação, devido ao número reduzido de parâmetros EOS extraídos; e $i$ ii) o elevado desempenho atingido pelo detector e classificador.

De forma a discutir a presente proposta, este artigo é organizado da seguinte maneira. Na seção 2, o sistema proposto é apresentado em detalhes. Na seção 3 são apresentados os resultados de detecção e classificação do mesmo. Finalmente, na seção 4 , as conclusões gerais sobre o sistema proposto são apresentadas.

\section{SISTEMA PROPOSTO}

O sistema proposto neste trabalho pode ser representado pelo diagrama em blocos da figura 1 , em que o primeiro bloco implementa o algoritmo para a detecção e o segundo bloco, 


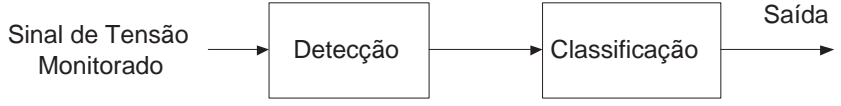

Figura 1: Diagrama de blocos do sistema proposto.
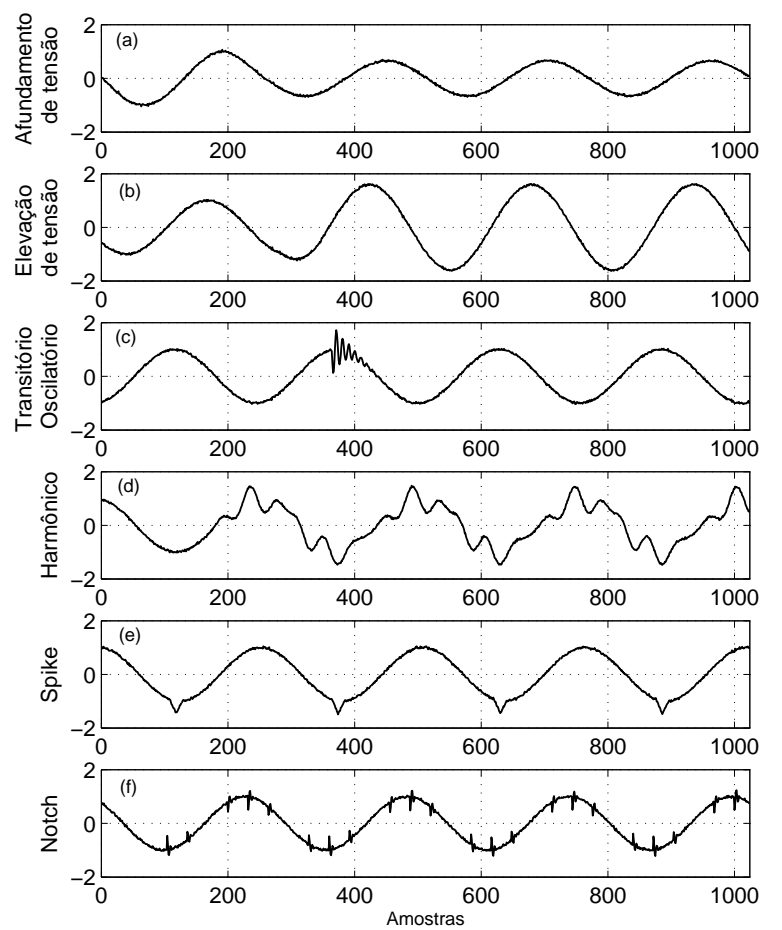

Figura 2: Exemplos de eventos de afundamento de tensão (a), elevação de tensão (b), transitório oscilatório (c), harmônico (d), spike (e) e notch (f).

que será ativado apenas quando algum tipo de distúrbio for detectado, implementa o algoritmo para a classificação dos distúrbios. É importante destacar que apenas os distúrbios que aparecem na forma de onda da tensão serão analisados.

O sistema será capaz de detectar e classificar as seguintes classes de distúrbios: afundamentos de tensão, elevações de tensão, transitórios oscilatórios, harmônicos, spikes e notches. A figura 2 ilustra um evento de cada uma destas classes.

O projeto do sistema pode ser divido em duas etapas, detecção e classificação, como descrito a seguir.

\subsection{Etapa de Detecção}

O diagrama de blocos do detector é apresentado na figura 3. A seguir os blocos deste diagrama são detalhados e os

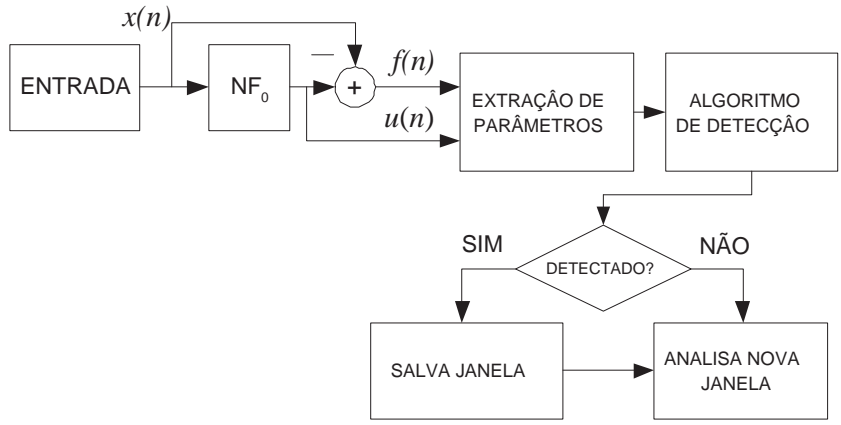

Figura 3: Diagrama de blocos da etapa de detecção.

equacionamentos são apresentados.

\section{i) Entrada:}

O bloco Entrada contém o vetor x com 256 amostras do sinal de entrada. O sistema foi projetado para uma freqüência de amostragem $\left(f_{s}\right)$ de $256 \times 60 \mathrm{~Hz}$. A escolha desta freqüência de amostragem é devido ao fato de que a faixa de freqüência de 0 a 7,64 kHz é suficiente para caracterizar a maioria dos distúrbios que ocorrem na forma de onda da tensão (Quality, 1995), muito embora outras frequiências de amostragem podem ser utilizadas.

\section{ii) NFo}

O bloco $N F_{0}$ implementa um filtro notch sintonizado na freqüência da componente fundamental $\left(f_{0}=60 \mathrm{~Hz}\right)$ de $x(n)$. Devido, principalmente, ao baixo custo computacional e a maior seletividade na freqüência de interesse, neste trabalho se utiliza uma estrutura de filtro IIR de $2^{\text {a }}$ ordem (Mitra, 2005), cuja transformada z é dada pela equação:

$$
H_{0}(z)=\frac{1+a_{0} z^{-1}+z^{-2}}{1+\rho_{0} a_{0} z^{-1}+\rho_{0}^{2} z^{-2}}
$$

onde

$$
a_{0}=-2 \cos \omega_{0}
$$

e $\rho_{0}$ é o fator notch, com $0 \ll \rho_{0}<1$.

A curva de resposta em magnitude deste filtro é mostrada na figura 4.

\section{iii) Extração de Parâmetros}

O bloco Extração de Parâmetros extrai os parâmetros de EOS do sinal pelo cálculo dos cumulantes dos vetores $\mathbf{u}$ e f , formados pelas amostras dos sinais $u(n)$, referente ao sinal de entrada sem a componente fundamental e $f(n)$ que é dado pela subtração de $u(n)$ ao sinal 


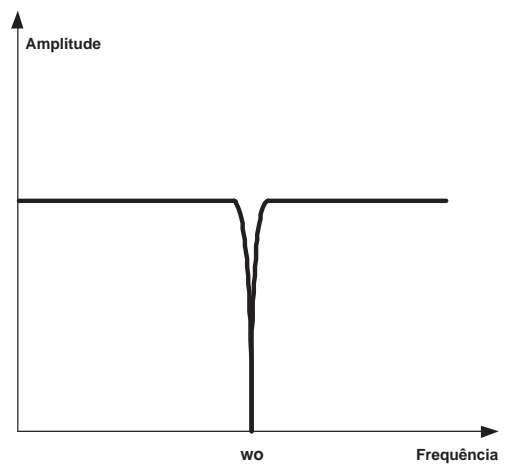

Figura 4: Resposta em freqüência de um filtro notch típico IIR, onde $\omega_{o}=2 \pi f_{0}$.

de entrada $x(n)$, referente à estimativa da componente fundamental.

Os parâmetros de EOS, e o número destes, podem ser escolhidos de forma a haver um melhor compromisso entre baixo custo computacional e desempenho. Neste trabalho, devido ao alcance de resultados melhores e satisfatórios, se utiliza dois parâmetros, um do vetor $\mathbf{f}$ e outro do vetor $\mathbf{u}$, cada qual de $2^{\mathrm{a}}$ ou de $4^{\mathrm{a}}$ ordem.

As expressões (equações) para os cumulantes de $2^{\mathrm{a}}$ e $4^{\mathrm{a}}$ ordens para um vetor $\mathbf{z}$ constituído de variáveis aleatórias, quando $E\{\mathbf{z}\}=0$, são expressas por:

$$
c_{2, z}(i)=E\{z(n) z(n+i)\},
$$

e

$$
c_{4, z}(i)=E\left\{z(n) z^{3}(n+i)\right\}-3 c_{2, z}(i) c_{2, z}(0),
$$

respectivamente, onde $i$ é o $i$-ésimo atraso, e $z(n)$ é o $n$-ésimo elemento do vetor $\mathbf{z}$ (Mendel, 1991).

Para sinais de comprimento $N$ finito, as expressões (3)(4) podem ser estimadas, como demonstrado em Ribeiro et al. (2006), por:

$$
\hat{c}_{2, z}(i):=\frac{1}{N} \sum_{n=0}^{N-1} z(n) z[\bmod (n+i, N)],
$$

$$
\begin{gathered}
\hat{c}_{4, z}(i):=\frac{1}{N} \sum_{n=0}^{N-1} z(n) z^{3}[\bmod (n+i, N)]- \\
\frac{1}{N^{2}} \sum_{n=0}^{N-1} z(n) z[\bmod (n+i, N)] \sum_{n=0}^{N-1} z^{2}(n)
\end{gathered}
$$

onde $\bmod (n+i, N)$ é o resto inteiro da divisão de $n+i$ por $N$.

Para a obtenção de um reduzido conjunto de parâmetros para a detecção e, posteriormente, para a classificação dos distúrbios, é necessária a utilização de alguma ferramenta que avalie a separação entre as diversas classes. Assim, decidiu-se usar o método FDR (Fisher Discriminat Ratio) pela sua simplicidade e obtenção de resultados satisfatórios (Theodoridis e Koutroumbas, 1999). A função custo do critério FDR é:

$$
\mathbf{J}_{c}=\left(\mathbf{m}_{1}-\mathbf{m}_{2}\right)^{2} \odot \frac{1}{\mathbf{D}_{1}^{2}+\mathbf{D}_{2}^{2}}
$$

onde $\mathbf{J}_{c}=\left[J_{1} \cdots J_{L_{l}}\right]^{T}, L_{l}$ é o número total de parâmetros, $\mathbf{m}_{1}$ e $\mathbf{m}_{2}$, e $\mathbf{D}_{1}^{2}$ e $\mathbf{D}_{2}^{2}$ são, respectivamente, os vetores de média e variância dos vetores de parâmetros $\mathbf{p}_{1, k}, \quad k=1,2, \cdots, M_{p}$ e $\mathbf{p}_{2, k}, \quad k=1,2, \cdots, M_{p}$, onde $M_{p}$ denota o número total de vetores. O símbolo $\odot$ refere-se ao produto de Hadamard $\mathbf{r} \odot \mathbf{s}=$ $\left[r_{0} s_{0} \cdots r_{L_{r}-1} s_{L_{r}-1}\right]^{T}$. O $i$-ésimo elemento do vetor de parâmetros dado por (7) tendo o maior valor, é escolhido para o uso nos métodos de detecção e classificação. Aplicando este procedimento para todos os elementos do vetor principal, os $K$ parâmetros associados com os $K$ maiores valores no vetor $\mathbf{J}_{\mathbf{c}}$ são usados pelo detector e classificador.

\section{iv) Algoritmo de Detecção}

Neste bloco, é utilizada uma técnica para detectar de forma eficiente a ocorrência de distúrbio através do processamento dos parâmetros extraídos do sinal. Para isso foi empregado o detector de Bayes baseado no critério ML (Maximum Likelihood) devido a sua simplicidade, facilidade de projeto e baixo custo computacional (Theodoridis e Koutroumbas, 1999).

Para esta aplicação, o problema de detecção pode ser visto como um problema de decisão entre duas hipóteses, uma hipótese $\mathcal{H}_{0}$ referente a condições normais do sinal (sinais sem distúrbio) e outra hipótese $\mathcal{H}_{1}$ referente a condições anormais do sinal (sinais com distúrbios).

Diversas técnicas de detecção lineares e não-lineares podem ser aplicadas para determinar a superfície de separação entre as hipóteses $\mathcal{H}_{0}$ e $\mathcal{H}_{1}$. Para a presente técnica, baseando-se na teoria de Bayes, a detecção pode ser feita através da inequação:

$$
\frac{p\left(\mathbf{x} \mid \mathcal{H}_{1}\right)}{p\left(\mathbf{x} \mid \mathcal{H}_{0}\right)} \lesseqgtr \frac{P\left(\mathcal{H}_{0}\right)}{P\left(\mathcal{H}_{1}\right)},
$$

onde $\mathbf{x}$ é um vetor constituído de elementos aleatórios, os quais são parâmetros extraídos do sinal de tensão, $P\left(\mathcal{H}_{i}\right)$ representa a probabilidade a priori e $p\left(\mathbf{x} \mid \mathcal{H}_{i}\right)$ representa a função densidade de probabilidade (pdf - probability density function) condicional de obter $\mathbf{x}$ dado que a classe é $\mathcal{H}_{i}$. A função densidade de probabilidade condicional utilizada é dada por: 


$$
p\left(\mathbf{x} \mid \mathcal{H}_{i}\right)=\frac{1}{(2 \pi)^{L / 2}\left|\sum_{i}\right|^{1 / 2}} e^{-\frac{1}{2}\left(\mathbf{x}-\mu_{i}\right)^{T} \sum_{i}^{-1}\left(\mathbf{x}-\mu_{i}\right)},
$$

onde $\mu_{i}=E\{\mathbf{x}\}$ é o vetor valor médio da classe $\mathcal{H}_{i}$, $\sum_{i}$ é a matriz de covariância da mesma classe definida por

$$
\sum_{i}=E\left\{\left(\mathbf{x}-\mu_{\mathbf{i}}\right)\left(\mathbf{x}-\mu_{\mathbf{i}}\right)^{T}\right\},
$$

e $\left|\sum_{i}\right|$ denota o determinante de $\sum_{i}$.

Supondo $P\left(\mathcal{H}_{0}\right)=P\left(\mathcal{H}_{1}\right)=1 / 2$ e as pdf's dadas por (9), o detector dado por (8) assume a forma:

$$
\frac{\left|\sum_{0}\right|^{\frac{1}{2}} e^{-\frac{1}{2}\left(\mathbf{x}-\mu_{\mathbf{b}}\right)^{T} \sum_{1}^{-1}\left(\mathbf{x}-\mu_{1}\right)}}{\left|\sum_{1}\right|^{\frac{1}{2}} e^{-\frac{1}{2}\left(\mathbf{x}-\mu_{\mathbf{a}}\right)^{T} \sum_{0}^{-1}\left(\mathbf{x}-\mu_{\mathbf{0}}\right)}} \gtreqless 1
$$

onde $\mu_{0}$ e $\mu_{1}$ são os vetores valor médio das classes $\mathcal{H}_{0}$ e $\mathcal{H}_{1}$ respectivamente, e $\sum_{0}$ e $\sum_{1}$ são as matrizes de covariância das respectivas classes.

A escolha dos valores das probabilidades a priori se deveu ao não conhecimento prévio das estatísticas da ocorrência dos distúrbios em sinais de tensão.

Sendo assim, a expressão a esquerda de (11) é aplicada ao vetor de parâmetros extraído, e se o valor encontrado for maior ou igual a 1 , o sinal é considerado com presença de distúrbio, caso contrário o mesmo é considerado como sinal sem distúrbio.

\section{v) Salva Janela}

O bloco Salva Janela armazena uma janela do sinal de erro $\{u(n)\}$ e da fundamental estimada $\{f(n)\}$ do distúrbio detectado, quando algum distúrbio é detectado. $\mathrm{O}$ algoritmo de detecção faz uma leitura, amostra por amostra, e detecta o início do distúrbio. O bloco Salva Janela armazena uma janela contendo um ciclo da fundamental antes do início do distúrbio e três ciclos após o início do distúrbio, alcançando um total de 1.024 amostras.

A figura 5 mostra um exemplo de um sinal de tensão com um distúrbio transitório oscilatório sendo monitorado. Na figura 6 são mostradas as janelas da fundamental estimada e do sinal de erro em 4 ciclos da fundamental quando a freqüência de amostragem $(f s)$ é $256 \times 60 \mathrm{~Hz}$ para o sinal mostrado na figura 5 .

\section{vi) Analisa Nova Janela}

O bloco Analisa Nova Janela apenas contém a informação de restabelecimento do monitoramento para propósitos de detecção.

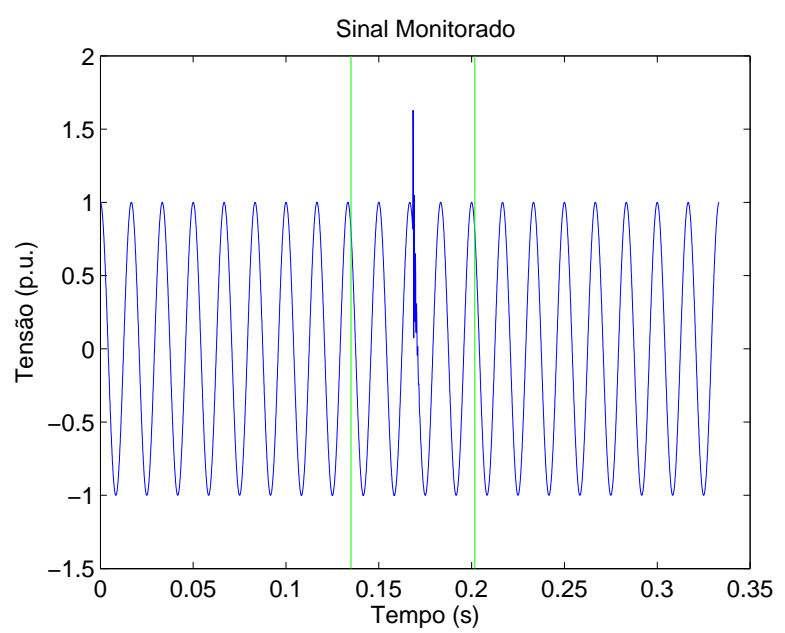

Figura 5: Exemplo de sinal de tensão com um distúrbio transitório oscilatório sendo monitorado.

\subsection{Etapa de Classificação}

$\mathrm{Na}$ etapa de detecção, anteriormente descrita, o algoritmo de detecção disponibiliza ao classificador, uma vez detectado um evento, a estimativa da fundamental $\{f(n)\}$ e o sinal de erro $\{u(n)\}$. Assim, na etapa de classificação, a extração de parâmetros é efetuada, inicialmente, no sinal de erro $\{u(n)\}$, e os parâmetros extraídos são apresentados ao classificador, conforme mostrado na figura 7 .

Como classificador, utilizou-se uma rede neural artificial (RNA) do tipo perceptron de múltiplas camadas (MLP multi-layer perceptron) (Haykin, 1999) (vide figura 8), que é uma estrutura de rede composta pela camada de entrada, uma ou mais camadas de neurônios ocultos, e a camada de saída. As RNA apresentam um custo computacional relativamente baixo na fase de aplicação quando comparado com outros classificadores não-lineares, como as máquinas de vetor de suporte (SVM - Support Vector Machine) por exemplo. Entretanto, as RNA têm apresentado bons resultados quando aplicadas à classificação de distúrbios de QEE, conforme mostrado em Cerqueira et al. (2005), Janik e Lobos (2006) e Ferreira et al. (2006).

Durante o projeto do sistema, observou-se que os sinais de erro referentes a um evento de afundamento de tensão e elevação de tensão possuíam características semelhantes , de modo que as EOS de tais sinais não foram capazes de distinguir os mesmos e, portanto, estes eventos foram agrupados em uma única classe. Uma nova metodologia, baseada apenas no valor RMS da estimativa da componente fundamental foi utilizada para separar tais classes. Dado que esta nova metodologia é bastante simples e apresenta um desempenho satisfatório (vide seção 3), houve a melhora no 

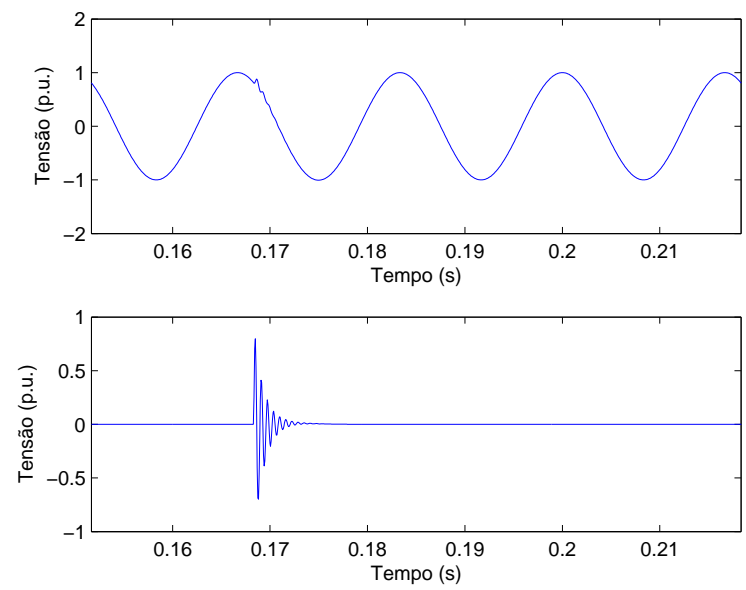

Figura 6: Janelas de 1024 amostras armazenadas pelo detector para o evento exemplificado na figura 5 .

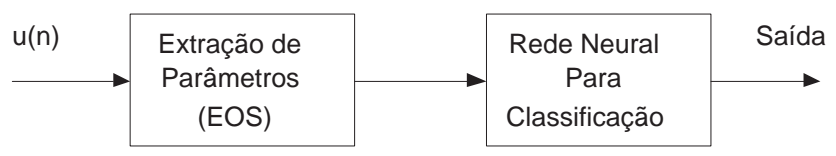

Figura 7: Diagrama de blocos do classificador apartir do sinal de erro $(u(n))$.

desempenho e a redução da complexidade da rede neural que efetua a classificação das outras classes, visto que tem uma classe a menos para classificar. Tal metodologia será abordada adiante. Desta maneira, o classificador mostrado na figura 7, que implementa uma rede neural, foi projetado para classificar 5 classes: afundamento/elevação de tensão, harmônicos, spikes, notches e transitórios oscilatórios.

O projeto do classificador é dividido em três principais etapas: extração de parâmetros, seleção de parâmetros e classificação.

Foi verificado em Ferreira et al. (2006) que os cumulantes de segunda ordem armazenam mais energia do sinal $(u(n))$ do que os cumulantes de terceira e quarta ordem e, portanto, são mais representativos e suficientes para a classificação de distúrbios. Assim, apenas os cumulantes de segunda ordem, obtidos conforme equação (5), foram utilizados como parâmetros na classificação dos distúrbios.

Após a extração de parâmetros, obtém-se um vetor $\hat{\mathbf{c}}_{2, u}$, referente aos cumulantes de segunda ordem com o mesmo número de amostras $(N)$ do sinal de erro $\{u(n)\}$.

Assim como no projeto do detector, o critério FDR (equação (7)) foi aplicado a cada uma das classes de distúrbios consideradas. Comparando uma classe com todas as

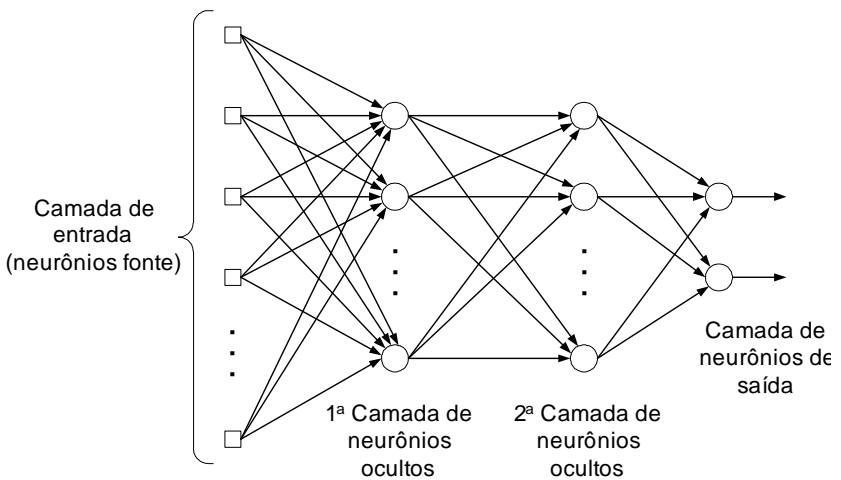

Figura 8: Arquitetura de um perceptron de múltiplas camadas.

outras, selecionou-se apenas um parâmetro com o maior valor de $\mathbf{J}_{c}$ de cada classe, resultando em um novo vetor de parâmetros agora contendo apenas 5 parâmetros, referentes aos cumulantes de segunda ordem mais representativos (que conduzem à uma melhor separabilidade entre classes) de cada classe. Este novo vetor de parâmetros é apresentado a uma rede neural artificial para a classificação.

É importante ressaltar que a seleção de parâmetros, tanto na etapa de detecção quanto na de classificação, é realizada apenas durante o projeto do sistema. Com o sistema em operação, apenas os 2 cumulantes (um de segunda ordem e um de quarta ordem), previamente selecionados, são extraídos para a detecção e 5 cumulantes de segunda ordem são extraídos para a classificação, o que implica em uma considerável redução da complexidade computacional e, conseqüentemente, leva a uma simplificação da rede neural.

Para o sistema de classificação aqui proposto, a rede MLP é composta por três camadas (apenas uma camada oculta), sendo cada camada composta por 5 neurônios. Os 5 neurônios na camada de entrada são referentes aos 5 parâmetros selecionados e os 5 neurônios da camada de saída (terceira camada) representam os 5 parâmetros de cada padrão de resposta apresentado a rede. Os padrões de resposta apresentados à rede foram os seguintes: yd $=[1-1-1-1-1]^{T}$ para transitórios oscilatórios, yd $=\left[\begin{array}{lll}-1 & 1-1-1-1\end{array}\right]^{T}$ para harmônicos, $\mathbf{y d}=\left[\begin{array}{lll}-1-1 & 1-1-1\end{array}\right]^{T}$ para afundamentos/elevações de tensão, yd $=\left[\begin{array}{lll}-1-1-1 & 1-1\end{array}\right]^{T}$ para notches e yd $=\left[\begin{array}{lll}-1-1-1-1 & 1\end{array}\right]^{T}$ para spikes. Desta forma, o critério adotado para definição da classe do evento foi o do neurônio com maior valor de saída. Os pesos e bias foram inicializados de acordo com o algoritmo de Nguyen e Widrow (1990). A função de ativação utilizada foi a tangente hiperbólica e o algoritmo de treinamento foi baseado na otimização de Levenberg-Marquardt (Haykin, 1999). 


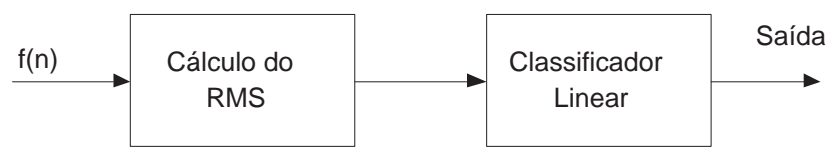

Figura 9: Diagrama de blocos do Classificador a partir do sinal $f(n)$.

Nas redes MLP, o sinal de entrada se propaga adiante, através da rede, camada por camada, até alcançar a camada de saída. Tipicamente, o treinamento de perceptrons de múltiplas camadas é realizado de forma supervisionada através de um algoritmo que se baseia na regra de aprendizagem por correção de erro. Este método é conhecido como algoritmo de retropropagação de erro (Haykin, 1999). A rede MLP tem sido bastante empregada recentemente (Cerqueira et al., 2005; Gaouda et al., 2002; Wang et al., 2001), uma vez que a mesma permite aproximar, com bom desempenho, as superfícies ótimas de separação entre as classes de distúrbios.

Para separar as classes de afundamentos e elevações de tensão, um segundo sistema foi implementado em que o classificador utiliza o valor médio quadrático (RMS - Root Mean Square) do sinal da estimativa da componente fundamental $\{f(n)\}$. Este segundo sistema está ilustrado na figura 9, e será acionado apenas quando o classificador principal (rede neural) apontar em sua saída a classe afundamento/elevação de tensão.

O valor RMS foi obtido de acordo com a equação 12.

$$
R M S(f)=\sqrt{\frac{\sum_{n=1}^{N}(f(n)-\bar{f})^{2}}{N-1}}
$$

Na equação (12), $f(n)$ representa um sinal discreto no tempo com $N$ amostras e $\bar{f}$ é a sua média. $N$ deve ser escolhido de modo que o somatório contenha múltiplos inteiros do período da componente fundamental.

Para o projeto do classificador linear (vide figura 9), calculou-se o valor RMS do conjunto de dados (previamente separados para treino) das classes de afundamentos e elevações de tensão, conforme equação (12). Assim, o número de amostras inicial $N$ de cada vetor referente a um distúrbio de afundamento ou elevação de tensão foi reduzido a uma amostra referente ao valor RMS do respectivo evento. Analisando as amostras obtidas, observou-se a existência de uma fronteira linear entre as classes de afundamentos e elevações de tensão, conforme ilustrado na figura 10.

Desta maneira, a fronteira linear ilustrada na figura 10 caracteriza o algoritmo que implementa o bloco Classificador Linear mostrado no diagrama de blocos da figura 9.

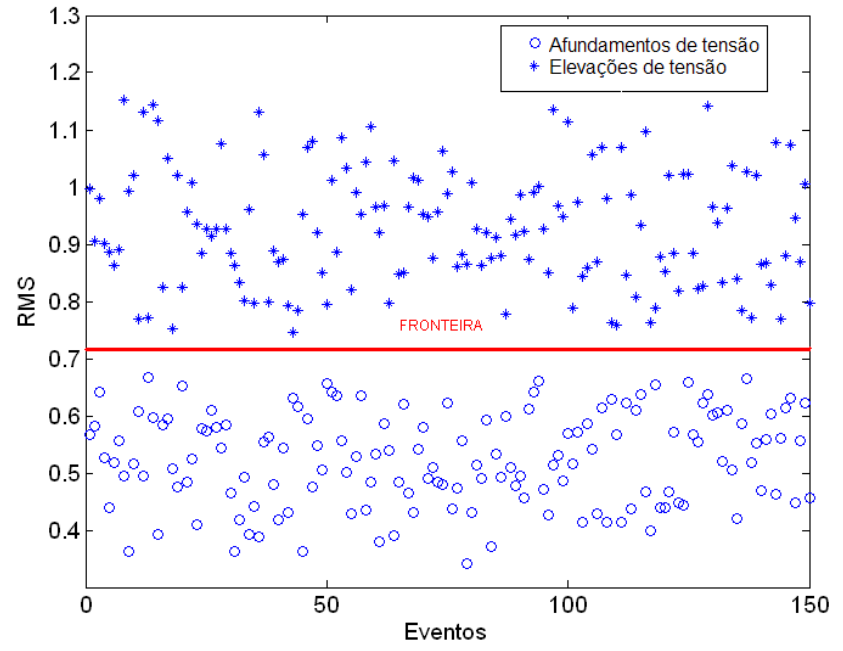

Figura 10: Fronteira linear que separa os eventos afundamento e elevação de tensão.

\section{RESULTADOS}

Para o projeto e validação do sistema proposto foram utilizados 3500 eventos simulados e divididos igualmente entre as seguintes classes: afundamentos de tensão, elevações de tensão, spikes, notches, harmônicos ${ }^{1}$, transitórios oscilatórios e sinais sem distúrbios. Estes sinais foram gerados com uma relação sinal ruído (SNR - Signal-to-Noise Ratio) de $30 \mathrm{~dB}$ utilizando os algoritmos propostos em Cerqueira et al. (2005) e seguindo as definições encontradas na norma do IEEE (Quality, 1995) e utilizadas na literatura, conforme Bollen (2000), Janik e Lobos (2006), Wang et al. (2001), Wang et al. (2004), Ribeiro et al. (2007) e Ferreira et al. (2006). Cumpre ressaltar que todos os resultados desta seção foram obtidos utilizando processamento off-line.

Com o objetivo de atingir um elevado índice de generalização no processo de detecção e classificação, as características de cada evento foram escolhidas de forma aleatória em intervalos pré-definidos. Dentro dos intervalos foi considerado que as características deveriam apresentar uma distribuição estatística uniforme, de maneira e evitar a ocorrência de um maior número de eventos com alguma característica semelhante. Os resultados de detecção do sistema proposto são mostrados na tabela 1 e os resultados de classificação podem ser vistos na tabela 2 .

É importante destacar as motivações que levaram à inclusão dos harmônicos como distúrbios a serem detectados e classificados, uma vez que, em geral, os harmônicos são tratados como eventos de regime permanente. São duas as motivações

\footnotetext{
${ }^{1}$ Para a classe de hamônicos foram simulados eventos contendo até o sétimo harmônico.
} 
para isto:

1. Existem situações em que o conteúdo harmônico de fato deve ser tratado como variante no tempo, como por exemplo, a energização de um transformador e o comportamento de inversores e conversores quando as cargas são variantes no tempo. Estas situações têm sido amplamente discutida dentro do IEEE Task Force in Harmonics e um trabalho recente sobre esta abordagem é apresentado em Duque et al. (2008).

2. Dependendo do ponto monitorado, várias cargas podem estar conectadas, sendo ligadas e desligadas em diversos momentos fazendo com que o conteúdo harmônico do mesmo apresente variações. Portanto, é importante que o algoritmo seja capaz de detectar estes distúrbios, uma vez que numa análise à posteriori poderá haver a identificação de algum mau funcionamento de equipamentos.

Uma comparação entre o método proposto e os métodos Optimal Time Frequency Resolution (OTFR) (Wang e Mamishev, 2004; Wang et al., 2004) e Low Complexity Event Classification (LCEC) (Cerqueira et al., 2005) foi realizada. A principal motivação para a escolha destes métodos para comparação está no fato de que ambos mostram bons resultados quando aplicados à detecção e classificação de distúrbios na forma de onda da tensão. É importante ressaltar também que os métodos OTFR e LCEC foram implementados e validados com os mesmos dados utilizados para validar o sistema proposto neste trabalho. Os resultados de tais métodos são também mostrados na tabela 2 . Note que a eficiência global é dada por

$$
\rho=\frac{1}{M_{d}} \sum_{i=1}^{M_{d}} C R_{i},
$$

onde $C R_{i}$ indica a razão de classificação e $M_{d}$ é o número de classes de distúrbios considerado.

Cumpre ressaltar que os resultados mostrados na tabela 2 foram obtidos considerando apenas distúrbios corretamente detectados.

Tabela 1: Taxas de acerto do algoritmo de detecção

\begin{tabular}{c|c}
\hline Detecção & $100 \%$ \\
\hline Falso Alarme & $0,2 \%$ \\
\hline
\end{tabular}

É importante enfatizar que como o detector obteve $0,2 \%$ (valor equivalente a um evento) de falso alarme, significa que o mesmo detectou um distúrbio em um sinal de tensão puro, isto é, desprovido de qualquer poluição relacionada a
Tabela 2: Taxas de classificação e eficiência global em \% obtida por LCEC, OTFR, e pelo método proposto.

\begin{tabular}{c||c|c|c} 
Evento & LCEC & OTFR & $\begin{array}{c}\text { Método } \\
\text { Proposto }\end{array}$ \\
\hline \hline Harmônicos & $95,6 \%$ & $\mathbf{1 0 0} \%$ & $\mathbf{1 0 0} \%$ \\
\hline $\begin{array}{c}\text { Afundamentos } \\
\text { de tensão }\end{array}$ & $99,6 \%$ & $\mathbf{1 0 0} \%$ & $\mathbf{1 0 0} \%$ \\
\hline $\begin{array}{c}\text { Elevações } \\
\text { de tensão }\end{array}$ & $99,6 \%$ & $\mathbf{1 0 0} \%$ & $\mathbf{1 0 0} \%$ \\
\hline Trans. Oscilatório & $\mathbf{1 0 0} \%$ & $97,8 \%$ & $\mathbf{1 0 0} \%$ \\
\hline Notch & $99,6 \%$ & $95,2 \%$ & $\mathbf{1 0 0} \%$ \\
\hline Spike & $99,0 \%$ & $99,6 \%$ & $\mathbf{1 0 0} \%$ \\
\hline \hline Eficiência Global & $98,9 \%$ & $98,7 \%$ & $\mathbf{1 0 0} \%$
\end{tabular}

algum distúrbio, encaminhando este sinal para o classificador. Como o classificador não foi projetado para classificar classes de sinais sem distúrbio, ele terá que apontar na saída uma das seis classes de distúrbios consideradas e, conseqüentemente, apontará um distúrbio que não ocorreu. Para este caso específico, o classificador apontou em sua saída um evento de spike. Portanto, a eficiência global do sistema de detecção e classificação proposto será dada pela eficiência global do detector, que foi de $99,8 \%$.

Uma forma de medir a complexidade computacional de um método com vistas a futuras implementações em DSP (Digital Signal Processor) é obter o número de operações matemáticas (miltiplicações e adições), tangentes hiperbólicas e exponenciais realizadas pelo algoritmo proposto na fase de operação. Assim, as tabelas 3 e 4 mostram tais operações para o método proposto. Note que os resultados de complexidade computacional são apresentados em duas etapas, detecção e classificação, e, portanto, a complexidade computacional total do sistema de detecção e classificação proposto é dada pela soma das complexidade de cada etapa.

Tabela 3: Complexidade computacional para o método de detecção proposto baseado em EOS.

\begin{tabular}{c||c} 
Evento & EOS \\
\hline \hline Adição & 2.822 \\
\hline Multiplicação & 2.835
\end{tabular}

A tabela 4 ilustra também a complexidade computacional dos métodos LCEC e OTFR, mostrando que o método proposto (EOS) obteve uma reduzida complexidade computacional quando comparado ao método OTFR e uma complexidade computacional levemente superior ao método LCEC. É importante ressaltar que os métodos LCEC e OTFR não 
Tabela 4: Complexidade computacional do método de classificação proposto baseado em EOS e dos métodos LCEC e OTFR.

\begin{tabular}{c||c|c|c} 
Evento & LCEC & OTFR & EOS \\
\hline \hline Adição & 6.162 & 86.373 & 5.175 \\
\hline Multiplicação & 1.974 & 108.022 & 5.180 \\
\hline $\exp ()$. & - & 70 & - \\
\hline $\tan ()$. & 22 & - & 10
\end{tabular}

propõem a detecção de distúrbios e, portanto, os respectivos resultados de complexidade computacional para a detecção não foram apresentados na tabela 3 .

Assim, o método proposto destaca-se pelo alto desempenho, conforme mostram as tabelas 1 e 2 , e pelo reduzido custo computacional, que foi obtido pelo número de operações matemáticas que o método realiza na fase de operação, conforme as tabelas 3 e 4 .

\section{CONCLUSÕES}

Neste trabalho, um sistema para detecção e classificação de distúrbios em QEE baseado em EOS para extração de parâmetros foi proposto. O uso de cumulantes de segunda e quarta ordem seguidos da razão de discriminação de Fisher para seleção de parâmetros levou a um pequeno número de parâmetros a serem usados pelos algoritmos de detecção e classificação, o que é uma das principais vantagens da técnica proposta. Esta vantagem sugere que o sistema proposto pode ser útil em aplicações em tempo real (on line) devido ao pequeno número de operações matemáticas necessárias na fase de operação.

É importante destacar também que o método proposto pode ser bastante útil em aplicações com alto nível de ruído, devido à imunidade dos parâmetros EOS utilizados. A eficiência das EOS em sistemas de monitoramento de QEE com altas taxas de ruído foi comprovada em Ribeiro et al. (2007).

Os resultados, em geral, mostram que o sistema proposto é apropriado para aplicações de QEE, apresentando índices de $100 \%$ de detecção com 0,2\% de falso alarme e uma eficiência global de $100 \%$ para a classificação das classes de distúrbios consideradas.

Como propostas futuras, pretende-se incorporar ao classificador a classe de sinais sem distúrbios, o que permitiria uma redundância maior no processo.

\section{AGRADECIMENTOS}

Ao Conselho Nacional de Desenvolvimento Científico e Tecnológico (CNPq), à Coordenação e Aperfeiçoamento de Pessoal de Nível Superior (CAPES) e à Fundação de Amparo à Pesquisa do Estado de Minas Gerais (FAPEMIG) pelo suporte financeiro.

\section{REFERÊNCIAS}

Abdel-Galil, T. K., Kamel, M., Youssef, A. M., El-Saadany, E. F. e Salama, M. M. A. (2004). Power Quality Disturbance Classification Using the Inductive Inference Approach, IEEE Transactions on Power Delivery 19(4): 1812-1818.

ANEEL (2008). PRODIST: Procedimentos de Distribuição, Technical report, www.aneel.gov.br.

Anil, K. J., Robert, P. W. e Jianchang, M. (2000). Statistical Pattern Recognition: A Review, IEEE Transactions on Pattern Analysis and Machine Intelligence 22(1).

Arrillaga, J., Bollen, M. H. J. e Watson, N. R. (2000). Power quality following deregulation, in Proc. 2000 of the IEEE 88(2): 246-261.

Bollen, M. H. J. (2000). Understanding Power Quality Problems Voltage Sags and Interruptions, IEEE Press.

Cerqueira, A. S., Duque, C. A., Trindade, R. e Ribeiro, M. (2005). Digital System for Detection and Classification of Electrical Events, IEEE International Symposium on Circuits and Systems, ISCAS pp. 5417-5420.

Duque, C. A., Ribeiro, M. V., Ramos, F. R. e Szczupak, J. (2005). Power Quality Event Detection Based on the Divide and Conquer Principle and Innovation Concept, IEEE Transactions on Power Delivery 20(4): 2361 2369.

Duque, C., Silveira, P., Baldwin, T. e Ribeiro, P. (2008). Novel method for tracking time-varying power harmonic distortions without frequency spillover, Power and Energy Society General Meeting - Conversion and Delivery of Electrical Energy in the 21st Century, 2008 IEEE pp. 1-6.

Ferreira, D. D., Cerqueira, A. S., Ribeiro, M. V. e Duque, C. A. (2006). HOS-based method for power quality event classification, 14th European Signal Processing Conference-EUSIPCO 2006.

Gaouda, A. M., Kanaun, S. H., Salama, M. A. e Chikhani, A. Y. (2002). Pattern Recognition Applications for Power System Disturbance Classification, IEEE Transactions on Power Delivery 17(3): 677-683. 
Haykin, S. (1999). Neural networks. A comprehensive foundation, 2 ed. edn, NJ: Englewood Cliffs, Prentice Hall.

He, H. e Starzyk, J. A. (2006). A Self-Organizing Learning Array System for Power Quality Classification Based on Wavelet Transform,, IEEE Transactions on Power Delivery 21(1): 286-295.

Heydt, G. T. (1998). Electric power quality: a tutorial introduction, IEEE Comput. Applicat. Power 11: 15-19.

Ibrahim, A. W. R. e Morcos, M. M. (2002). Artificial intelligence and advanced mathematical tools for power quality applications: a survey, IEEE Trans. on Power Delivery 17(2): 668-673.

Janik, P. e Lobos, T. (2006). Automated Classification of Power-Quality Disturbances Using SVM and RBF Networks, IEEE Transaction on Power Delivery 21(3): 1663 - 1669.

Mendel, J. M. (1991). Tutorial on higher-order statistics (spectra) in signal processing and system theory: theoretical results and some applications, Proc. of the IEEE 79(3): 278-305.

Mitra, S. K. (2005). Digital Signal Processing: A computerbased approach, third edn, McGraw-Hill.

Nguyen, D. e Widrow, B. (1990). Improving the learning speed of 2-layer neural networks by chossing initial values of the adaptive weights, IJCNN International Joint Conference on Neural Networks, Vol. 3, pp. 21-26.

Nikias, C. L. e Petropulu, A. P. (1993). Higher-Order Spectra Analysis - A Nonlinear Signal Processing Framework, Englewood Cliffs, Prentice Hall, NJ.

Oleskovicz, M., Coury, D. V., Carneiro, A. A. F. M., Arruda, E. F., F., O. D. e Souza, S. A. (2006). Estudo comparativo de ferramentas modernas de análise aplicadas à qualidade da energia elétrica, Sba: Controle \& Automação Sociedade Brasileira de Automatica 17(3): ISSN 0103-1759.

Poisson, O., Rioual, P. e Meunier, M. (2000). Detection and measurement of power quality disturbances using wavelet transform, IEEE Transactions on Power Delivery 15(3): 1039-1044.

Quality, I. S. C. C. . P. (1995). IEEE recommended practice for monitoring electric power quality, Technical report, IEEE.

Ramos, F. R., Ribeiro, M. V., Romano, J. M. T. e Duque, C. A. (2002). On signal processing approach for event detection and compression applied to power quality evaluation, in Proc. IEEE 10th International Conference on Harmonic and Quality of Power pp. 133-138.
Ribeiro, M. V., Marques, C. A. G., Duque, C. A., Cerqueira, A. S. e Pereira, J. L. R. (2006). Power Quality Disturbances Detection Using HOS, IEEE Power Engineering Society General Meeting .

Ribeiro, M. V., Marques, C. G., Cerqueira, A. S., Duque, C. A. e Pereira, J. L. R. (2007). Detection of Disturbances in Voltage Signals for Power Quality Analysis Using HOS, EURASIP Journal on Applied Signal Processing 2007: 13-25.

Santoso, S., Grady, W. M., Powers, E. J., Lamoore, J. e Bhatt, S. C. (2000). Characterization of distribution power quality events with Fourier and wavelet transforms, IEEE Transactions on Power Delivery 15(1): 247-254.

Theodoridis, S. e Koutroumbas, K. (1999). Pattern Recognition, Academic Press, San Diego.

Wang, M. e Mamishev, A. V. (2004). Classification of Power Quality Events Using Optimal Time-Frenquency Representations - Part 1: Theory, IEEE Transactions on Power Delivery 19(3): 1488-1495.

Wang, M., Ochenkowski, P. e Mamishevv, A. V. (2001). Classification of Power Quality disturbances using time-frequency ambiguity plane and neural networks, IEEE Power Eng. Soc. Summer Meeting 2: 1246-1251.

Wang, M., Rowe, G. I. e Mamishevv, A. V. (2004). Classification of Power Quality Events Using Optimal TimeFrequency Representations - Part 2: Application, IEEE Transactions on Power Delivery 19(3): 1496-1503. NJ.

Yang, H.-T. e Liao, C.-C. (2001). A de-noising scheme for enhancing wavelet-based power quality monitoring system, IEEE Transactions on Power Delivery 16(3): 353-360. 